\title{
Glutamine to proline conversion is associated with response to glutaminase inhibition in breast cancer
}

\author{
Maria T. Grinde ${ }^{*^{*}}$ (D) Bylgja Hilmarsdottir ${ }^{2,3}$, Hanna Maja Tunset ${ }^{1}$, Ida Marie Henriksen ${ }^{4}$, Jana Kim ${ }^{1,5}$, \\ Mads H. Haugen², Morten Beck Rye ${ }^{6,7}$, Gunhild M. Mælandsmo ${ }^{2,8}$ and Siver A. Moestue ${ }^{9,10}$
}

\begin{abstract}
Introduction: Glutaminase inhibitors target cancer cells by blocking the conversion of glutamine to glutamate, thereby potentially interfering with anaplerosis and synthesis of amino acids and glutathione. The drug CB-839 has shown promising effects in preclinical experiments and is currently undergoing clinical trials in several human malignancies, including triple-negative breast cancer (TNBC). However, response to glutaminase inhibitors is variable and there is a need for identification of predictive response biomarkers. The aim of this study was to determine how glutamine is utilized in two patient-derived xenograft (PDX) models of breast cancer representing luminal-like/ ER+ (MAS98.06) and basal-like/triple-negative (MAS98.12) breast cancer and to explore the metabolic effects of CB839 treatment.
\end{abstract}

Experimental: MAS98.06 and MAS98.12 PDX mice received CB-839 $(200 \mathrm{mg} / \mathrm{kg})$ or drug vehicle two times daily p.o. for up to 28 days ( $n=5$ per group), and the effect on tumor growth was evaluated. Expression of 60 genes and seven glutaminolysis key enzymes were determined using gene expression microarray analysis and immunohistochemistry $(\mathrm{HC})$, respectively, in untreated tumors. Uptake and conversion of glutamine were determined in the PDX models using HR MAS MRS after i.v. infusion of $\left[5^{13} \mathrm{C}\right]$ glutamine when the models had received CB-839 (200 mg/ $\mathrm{kg}$ ) or vehicle for 2 days ( $n=5$ per group).

Results: Tumor growth measurements showed that CB-839 significantly inhibited tumor growth in MAS98.06 tumors, but not in MAS98.12 tumors. Gene expression and IHC analysis indicated a higher proline synthesis from glutamine in untreated MAS98.06 tumors. This was confirmed by HR MAS MRS of untreated tumors demonstrating that MAS98.06 used glutamine to produce proline, glutamate, and alanine, and MAS98.12 to produce glutamate and lactate. In both models, treatment with CB-839 resulted in accumulation of glutamine. In addition, CB-839 caused depletion of alanine, proline, and glutamate ([1-13C] glutamate) in the MAS98.06 model.

Conclusion: Our findings indicate that TNBCs may not be universally sensitive to glutaminase inhibitors. The major difference in the metabolic fate of glutamine between responding MAS98.06 xenografts and non-responding MAS98.12 xenografts is the utilization of glutamine for production of proline. We therefore suggest that addiction to proline synthesis from glutamine is associated with response to CB-839 in breast cancer.

Keywords: ${ }^{13} \mathrm{C}$ MRS, Aldehyde dehydrogenase 18 family member A1 (ALDH18A1), Cancer treatment, CB-839, Gene expression analysis, Glutaminase, Glutaminase inhibitor, High-resolution magic angle spinning MR spectroscopy (HR MAS MRS), Immunohistochemistry, Patient-derived xenograft (PDX)

\footnotetext{
* Correspondence: maria.t.grinde@ntnu.no

1 Department of Circulation and Medical Imaging, Norwegian University of

Science and Technology (NTNU), 7489 Trondheim, Norway

Full list of author information is available at the end of the article
}

(c) The Author(s). 2019 Open Access This article is distributed under the terms of the Creative Commons Attribution 4.0 International License (http://creativecommons.org/licenses/by/4.0/), which permits unrestricted use, distribution, and reproduction in any medium, provided you give appropriate credit to the original author(s) and the source, provide a link to the Creative Commons license, and indicate if changes were made. The Creative Commons Public Domain Dedication waiver (http://creativecommons.org/publicdomain/zero/1.0/) applies to the data made available in this article, unless otherwise stated. 


\section{Introduction}

Alterations of metabolic pathways are a cancer hallmark, resulting in dependencies on specific nutrients for cell proliferation and tumor growth $[1,2]$. In addition to glucose, mammalian cells use glutamine to feed the tricarboxylic acid (TCA) cycle as an alternative source of carbon, and a precursor for proteins, lipids, and nucleic acids. Glutamine is also a key precursor in the synthesis of the antioxidant glutathione, which is important in maintaining the redox balance in cells and tissues [3]. Furthermore, glutamine can be converted via glutamate to proline, which is found to play an important regulatory role in cancer $[4,5]$. Recent research has identified proline as an important metabolite during adaption to hypoxia [6].

Although glutamine is a nonessential amino acid that can be synthesized from glutamate, many cancer cells depend on exogenous glutamine supply for proliferation and tumor growth $[7,8]$. Upregulated glutaminolysis is observed in many aggressive forms of human cancer, including colorectal cancer [9], gliomas [10], pancreatic cancer [11], melanoma [12], and breast cancer [13, 14], which highlights the importance of this amino acid in tumor metabolism.

The rate-limiting step in glutamine metabolism is the conversion of glutamine to glutamate, which is catalyzed by the enzyme glutaminase [15]. Glutaminase exists in several tissue-specific variants, encoded by two genes in mammals, kidney-type glutaminase (GLS1), and liver-type glutaminase (GLS2). GLS1 plays a central role in tumorigenesis, whereas the role of GLS2 in cancer remains unclear. GLS1 has been found to be higher expressed in TNBC compared to other subgroups of breast cancer [14] and is essential for the survival of TNBC cells with a deregulated glutaminolysis pathway [16].

Inhibition of glutaminase has been stated as an attractive therapeutic approach in various cancers $[8,13,14]$. The glutaminase inhibitor CB-839 (Calithera) is currently being tested in clinical trials for several malignancies including breast cancer (clinicaltrials.gov, ID: NCT02071862). CB-839 is found to be specific to GLS1 and not to GLS2 [14]. Gross and colleagues found that TNBC cell lines displayed a higher sensitivity to CB-839 compared to ER+ cell lines [14]. In addition, CB-839 caused a significant antitumor activity in two selected xenograft models representing TNBC and basal-like/ HER2+ breast cancer. In a clinical phase I trial, two of nine patients with TNBC treated with CB-839 experienced stabilized disease [17]. While these studies have shown promising results in the TNBC subgroup, there is a need to identify better predictive response biomarkers for optimal utilization and selection of patients that more likely will respond to CB-839.

The overall aim of this study was to identify metabolic characteristics associated with response to glutaminase inhibitors in breast cancer. First, we showed that CB-839 treatment has a differential effect on the growth of two patient-derived xenograft (PDX) models of breast cancer representing luminal-like, ER+ (MAS98.06) and basal-like, triple-negative (MAS98.12) breast cancer. Then, we investigated the potential causes of this differential response by (i) assessing the expression of selected genes and proteins directly involved in glutamine metabolism on tumor tissue from untreated mice and (ii) measuring downstream glutamine metabolites in tumor samples from CB-839-treated and untreated mice after administration of $\left[5-{ }^{13} \mathrm{C}\right]$ glutamine using ${ }^{13} \mathrm{C}$ high-resolution magic angle spinning MR spectroscopy (HR MAS MRS).

\section{Materials and methods Animal models}

The MAS98.06 and MAS98.12 patient-derived breast cancer xenograft models were established at the Institute of Cancer Research, Oslo University Hospital, as previously described [18]. The models have previously been classified as luminal B and basal-like molecular subtypes, respectively [19]. MAS98.12 has a triple-negative phenotype, whereas MAS98.06 is estrogen- and progesterone-receptor positive, and strongly dependent on estradiol supplement for tumor growth $[19,20]$. Tumor tissue was bilaterally and orthotopically transplanted into 5- to 6-week-old (18-20 g) female Hsd:Athymic Nude-Foxn $1^{\text {nu }}$ mice. The animals were kept under pathogen-free conditions at a temperature between 19 and $22^{\circ} \mathrm{C}$, humidity between 50 and 60\%, 20 air changes/h, and a 12-h light/dark cycle. The animals were fed RM1 diet (Scanbur BK, Karlslunde, Denmark) and distilled tap water ad libitum. The drinking water was supplemented with $17-\beta$-estradiol at a concentration of $4 \mu \mathrm{g} / \mathrm{ml}$ in order to replicate the conditions described in [18]. An overview of the experimental design is shown in Additional file 1.

All procedures and experiments involving animals were approved by the Norwegian Animal Research Authority (FOTS ID: 7713 and 9126) and carried out according to the European Convention for the Protection of Vertebrates used for Scientific Purposes.

\section{Tumor growth inhibition}

Following bilateral transplantation, mice carrying MAS98.06 and MAS98.12 tumors (experiment 1) were kept until tumor volume reached approximately $60 \mathrm{~mm}^{3}\left(60.3 \mathrm{~mm}^{3} \pm\right.$ $\left.32.4 \mathrm{~mm}^{3}\right)$. Mice from both models were randomized to receive either CB-839 $(200 \mathrm{mg} / \mathrm{kg})$ or drug-free vehicle two times per day for up to 28 days ( $n=5$ per group). CB-839 (Calithera Biosciences, CA, USA) was dissolved in 10\% cyclodextrin/saline solution and administered orally per gavage. Tumor length $(\mathrm{l})$ and width $(\mathrm{w})$ were measured using a 
digital caliper, and tumor volumes were calculated using the formula $V=\frac{1}{6}\left(\pi \times l \times w^{2}\right)$. The mice were sacrificed at day 14 (MAS98.12) and day 28 (MAS98.06) when untreated tumors approached the upper volume limit. Untreated tumors were collected for histopathological examination and HR MAS NMR (natural abundance) analysis.

\section{Gene expression analysis}

Gene expression analysis was performed (experiment 2) using previously generated and published data [21]. Briefly, RNA was isolated from tumor tissue from six animals from each xenograft models and hybridized to $4 \times$ 44k Agilent Whole Human Genome Oligo Microarrays according to the manufacturer's protocol. The microarray data was normalized and analyzed using $\mathrm{R}(\mathrm{v}$ 2.9.0) and the LIMMA Bioconductor package [22], normalized and $\log _{2}$ transformed. The microarray data is accessible through GEO Series accession number GSE37543. A total of 60 genes were selected for analysis, based on the KEGG maps central carbon metabolism in cancer (map 05230), arginine and proline biosynthesis (M00330), and glutathione biosynthesis (M00118) [23], which outline potential metabolic fates of glutamine in human cells.

Normalized and $\log _{2}$-transformed data was imported into Qlucore Omics Explorer 3.3 (Qlucore AB, Lund, Sweden) for statistical analysis. Testing for differential expression of genes between the xenograft models was performed using $t$ tests with Empirical Bayesian correction of the test statistics [22]. To account for multiple testing, an adjusted $q$ value of 0.05 (using Benjamini \& Hochberg's false discovery rate) was defined as the threshold for statistical significance [24].

The heatmap was generated in $\mathrm{R}$ ( $\mathrm{v}$ 3.3.2) using RStudio ( $\mathrm{v}$ 1.1.447). Hierarchical clustering was performed using the in-house made R-package Clustermap [25]. In brief, median-centered and $\log _{2}$-transformed RPPA data were clustered using Euclidean distance and complete linkage. For heatmap visualization of the data, values are normalized to the range $[-1,1]$ by application of a nonlinear sigmoid transformation $f(x)=\tanh (x)$. This limits the visual dominance of outlier values while maintaining the order of the values, since $f$ is strictly increasing.

To determine whether the metabolic characteristics of MAS98.06 and MAS98.12 xenografts are representative of the luminal B and basal-like subtypes of breast cancer, respectively, we accessed a previously published gene expression data set that in total includes 19 basal-like and 7 luminal B PDX models [19]. Gene expression of SLC1A5, GLS1, GLUL, and GLUD1 was accessed and are displayed as waterfall plots in Additional file 2. The microarray data is available at the Gene Expression Omnibus (GEO) with accession number GSE44666.

\section{Immunohistochemistry IHC staining}

Seven proteins (ALDH18A1, GLS1, GLUD1, GS, Myc, PYCR1, SLC1A5) were selected for protein expression analysis based on prior knowledge on their relevance in glutaminolysis. Firstly, glutamine transporters ensure uptake of glutamine into the cells, of which neutral amino acid transporter $\mathrm{B}(0)$ (coded by the gene SLC1A5 (Solute Carrier Family 1 Member 5), hereby abbreviated as SLC1A5) has received high attention since it has been shown that increased expression correlates with poor patient prognosis in many cancer types [9, 26-28]. Glutamine synthetase (GS) is the enzyme that catalyzes the conversion from glutamate to glutamine. Absence of GS is correlated with high GLS1 activity and can be associated with glutamine addiction in invasive and aggressive breast cancer phenotypes [29, 30]. Some cancer cells also rely on glutamate dehydrogenase 1 (GLUD1)-mediated Glu deamination to fuel the TCA cycle [31]. Glutamine can be converted to proline via glutamate, and the enzymes pyrroline-5-carboxylate reductase 1 (PYCR1) and aldehyde dehydrogenase 18 family member A1 (ALDH18A1) are found to be key enzymes in the conversion $[6,32,33]$. The proto-oncogene Myc is shown to be a key regulator of glutaminolysis affecting glutamine uptake, GLS1 activity, and proline metabolism [4, 26, 34-36].

Tumors from untreated mice (experiment 1) were fixed in $10 \%$ neutral-buffered formalin and embedded in paraffin. Information about primary antibodies are presented in a table in Additional file 3. GLUD1, SLC1A5, ALDH18A1, and PYCR1 staining were performed at the Cellular \& Molecular Imaging Core Facility (CMIC), NTNU, Norway. GLS1, GS, and cMYC staining were performed at Covance Laboratories Inc., (Greenfield, USA). The following protocols were applied: Tumor specimens were cut into $4 \mu \mathrm{m}$ sections which were dried, deparaffinized, and rehydrated. Heat-induced antigen retrieval was then performed for 10 (Covance, Antigen Retrieval Solution, Leica, AR9661) or 20 (CMIC, Target Retrieval Solution: Dako, low pH 6, K8005) minutes. Endogenous peroxidase activity was quenched with peroxidase block $\left(\mathrm{H}_{2} \mathrm{O}_{2}\right)$. Sections were incubated with primary antibodies (Additional file 3) for 15 (Covance) or 40 (CMIC) mins in room temperature. Immunohistochemical reactions were visualized as specified by the vendor using either (Covance) Dako Rabbit Envision+HRP with DAB+ for use with rabbit primary antibodies (K4011, Dako) or (CMIC) Bond PDAB Reagent Kit (Leica DS9800). Sections were counterstained with hematoxylin.

\section{IHC evaluation}

Immunohistochemical markers were evaluated by a semiquantitative approach used to assign the histo-score 
( $\mathrm{H}$-score) for each tumor. The $\mathrm{H}$-score is given as the sum of the percentage of staining multiplied by an ordinal value corresponding to the intensity level $(0=$ negative, $1=$ weak, $2=$ moderate, $3=$ strong $)$ : $[1 \times(\%$ cells $1+)+2 \times(\%$ cells $2+)+3 \times(\%$ cells $3+)]$. The final score, ranging from 0 to 300 , gives more relative weight to higher intensity labeling in a given tumor sample [24, 37, 38].

An experienced pathologist from Covance Inc. scored slides labeled with antibodies against GLS1, GS, and cMYC. Two researchers (MTG and SAM) scored each slide from GLUD1, SLC1A5, ALDH18A1, and PYCR1 independently in a blinded manner. Final $\mathrm{H}$-score is mean $\mathrm{H}$-score $\pm \mathrm{SD}$ from the two researchers. Some MAS98.06 tumors had central necrosis, and areas with necrosis were excluded from the analysis.

\section{Glutamine metabolism}

Another group of mice (experiment 3) carrying MAS98.06 $\left(n=12,30.0 \pm 10.3 \mathrm{~mm}^{2}\right)$ or MAS98.12 $(n=$ $11,41.4 \pm 10.6 \mathrm{~mm}^{2}$ ) were randomly distributed to receive CB-839 or drug-free vehicle for 2 days as described above. Three hours after the final treatment, animals received an intravenous infusion of ${ }^{13} \mathrm{C}$-enriched glutamine (99\% enrichment, Cambridge Isotope Laboratories) while under isoflurane anesthesia, as described in [39]. The mice received $1.2 \mathrm{mg}\left[5^{-13} \mathrm{C}\right]$ glutamine/g body weight, dissolved in sterile PBS. A 3-min bolus of 0.3 $\mathrm{mg} / \mathrm{g}$ body weight was followed by continuous infusion of $0.005 \mathrm{mg} / \mathrm{g}$ body weight/min for $180 \mathrm{~min}$, in a total infusion volume of 0.8 to $1.1 \mathrm{ml}$ (depending on body weight). The mice were sacrificed, and tumor tissue samples were collected, snap frozen, and stored in liquid nitrogen until NMR analysis.

\section{NMR experiments}

\section{NMR spectroscopy of tumor tissue samples}

Tumor samples from both ${ }^{13} \mathrm{C}$ glutamine-labeled $(n=5$ to six per group, experiment 3) and unlabelled (N.A.: natural abundance, $n=3$ per group, experiment 1) tumors $(39.9 \pm 1.1 \mathrm{mg})$ were cut to fit into a $50-\mu \mathrm{l}$ zirconium HR MAS rotor (4-mm diameter). Lock reference containing $\mathrm{D}_{2} \mathrm{O}$ with formate $(25 \mathrm{mM})$ was added to the rotor $(\sim 16 \mu \mathrm{l})$. The HR MAS MR spectra were recorded using a Bruker Advance DRX600 spectrometer (14.1 T) (Bruker Biospin $\mathrm{GmbH}$, Germany) containing a ${ }^{1} \mathrm{H} /{ }^{13} \mathrm{C}$ MAS probe. Samples were spun at $5 \mathrm{kHz}$ at magic angle, and the temperature was kept at $4{ }^{\circ} \mathrm{C}$ during the whole experiment. NMR spectra were acquired using the following NMR sequences and acquisition parameters: One-dimensional ${ }^{1} \mathrm{H}$ NOESY pulse sequence with water presaturation (Bruker; noesygppr1d). Acquisition time was $2.7 \mathrm{~s}$, repetition time $6.7 \mathrm{~s}$, sweep width was $30 \mathrm{ppm}$, and 128 scans were acquired. The ${ }^{13} \mathrm{C}$ MR spectra were acquired using a single pulse experiment, with ${ }^{1} \mathrm{H}$ decoupling applied during recycle delay and acquisition (Bruker; zgpg30). The flip angle was $30^{\circ}$, acquisition time $0.9 \mathrm{~s}$, repetition time $1.9 \mathrm{~s}$, sweep width $250 \mathrm{ppm}$, and $16 \mathrm{k}$ scans were obtained. Total acquisition time per sample was $9 \mathrm{~h}$.

\section{Analysis of NMR spectra}

NMR spectra were Fourier transformed after application of line broadening $\left({ }^{1} \mathrm{H}: 0.3 \mathrm{~Hz},{ }^{13} \mathrm{C}: 1 \mathrm{~Hz}\right.$ for tumors), and the chemical shift scale was calibrated to a reference peak ${ }^{1} \mathrm{H}$ : alanine at $1.48 \mathrm{ppm},{ }^{13} \mathrm{C}:\left[5^{-13} \mathrm{C}\right]$ glutamine $\left(\left[5-{ }^{13} \mathrm{C}\right] \mathrm{Gln}\right)$ at $180.4 \mathrm{ppm}$.

For quantification of tumor metabolites from ${ }^{1} \mathrm{H}$ NMR spectra, ERETIC2 (Bruker), which is based on PULCON (PULse length-based CONcentration determination), was applied [40]. Each spectrum was scaled to sample mass, and a total of four metabolites (alanine, glutamate, glutamine, and lactate) were quantified (relative) using Chenomx software (version 8.1, Alberta, Canada). Tumor ${ }^{13} \mathrm{C}$ NMR spectra were analyzed using MATLAB R2017a (The Mathworks, Inc., USA). Baseline correction was applied using an asymmetric least squares algorithm [41], and the NMR spectra were scaled to NMR sample mass. For determination of ${ }^{13} \mathrm{C}$-enriched peaks in tumors, 20 relevant peaks from the ${ }^{13} \mathrm{C}$ NMR spectra were selected and integrated, and peaks that were significantly higher (Student's $t$ test, $p<0.05)$ in $\left[5^{-13} \mathrm{C}\right]$ labeled compared to natural abundance tumors were considered ${ }^{13} \mathrm{C}$ enriched. The amount of eight ${ }^{13} \mathrm{C}$-labeled metabolites in the tumors were calculated by subtracting natural abundance spectra from ${ }^{13} \mathrm{C}$-enriched spectra. The results are shown in Additional file 4 (panel c).

The different experimental groups $\left({ }^{13} \mathrm{C}\right.$-enriched controls versus natural abundance controls from both models, ${ }^{13} \mathrm{C}$-enriched CB-839-treated versus ${ }^{13} \mathrm{C}$-enriched controls from both models, and ${ }^{13} \mathrm{C}$-enriched MAS98.06 controls versus ${ }^{13} \mathrm{C}$-enriched MAS98.12 controls) were compared statistically using Student's $t$ test. $P$ values less than 0.05 were considered statistically significant.

\section{Results \\ CB-839 inhibits tumor growth in the luminal-like (MAS98.06)}

Tumor growth curves from CB-839-treated and untreated MAS98.06 and MAS98.12 tumors (experiment 1) are displayed in Fig. 1 and show that CB-839 significantly inhibited the growth rate in MAS98.06 tumors. In treated mice, the tumor volume at day 28 was $28 \%$ of the volume of untreated controls $(p=0.0007)$. For the MAS98.12 model, no significant growth inhibition was observed.

\section{Expression of genes involved in glutaminolysis}

In untreated tumors (experiment 2), we observed that 31 genes were significantly higher expressed in 
MAS98.06

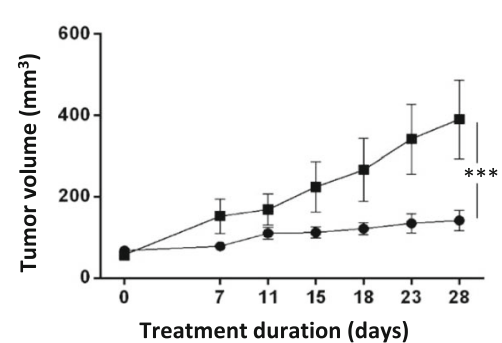

MAS98.12

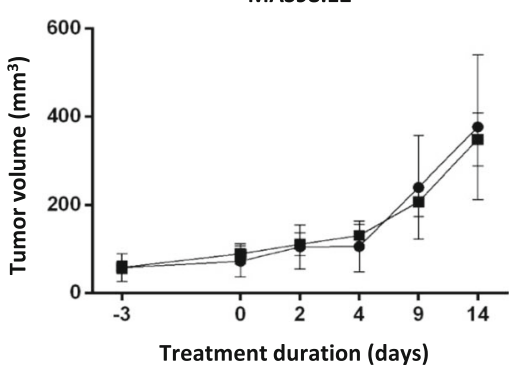

Fig. 1 Effect of CB-839 on tumor growth in luminal-like (MAS98.06) and basal-like (MAS98.12) PDX models. CB-839 significantly ( $p<0.001)$ reduces tumor growth in MAS98.06 tumors. Tumor growth of MAS98.12 breast cancer xenografts is not affected by CB-839. Mean \pm SEM values are plotted. $N=5$ for each group

MAS98.06 than in MAS98.12 tumors, of which 12 had at least a two-fold higher expression. For the MAS98.12 tumors, we observed that 17 genes were significantly higher expressed in this model compared to MAS98.06 tumors, of which 11 had at least a two-fold higher expression. The expression of all genes, as well as a schematic overview of genes that have at least a two-fold different expression between the models is presented in Fig. 2. A full overview of genes, expression levels and $q$ values is given as Additional file 5. Among the seven key enzymes that were selected for IHC analysis, we observed that the gene expression of GLUL and SLC1A5, as well as the important genes responsible for conversion between glutamate and proline, i.e., ALDH18A1 and PYCR1, were significantly more highly expressed in the responding MAS98.06 model. Contrarily, GLS1 and $M Y C$ were significantly more highly expressed in MAS98.12 tumors. Of note, SLC38A2 had two probes and the expression from both are displayed in the figure and table.

The expression of SLC1A5, GLS1, GS, and GLUD1 was compared across 19 basal-like and 7 luminal B PDX models (Additional file 2). Within both basal-like and luminal B models, a considerable variability (generally five-fold to ten-fold difference) in the expression of these key genes was observed.

\section{Key proteins involved in glutaminolysis are differentially expressed in the two models}

For better understanding of the differences in glutamine utilization between the models (experiment 1), we performed immunohistochemical (IHC) analysis on selected proteins involved in glutamine metabolism in untreated tumors. Figure 3 shows percentage areas with different DAB intensity for each antibody and model in addition to representative images. Using $\mathrm{H}$-score as an indicator of protein expression in the two xenograft models, MAS98.06 tumors had significantly higher expression of the two important enzymes responsible for conversion from glutamate to proline, i.e., ALDH18A1 $(p<1 \mathrm{e}-5)$, and PYCR1 $(p<0.05)$ in addition to GLUD1 $(p<0.05)$. They also had a significantly lower expression of SLC1A5 $(p<0.001)$ compared to MAS98.12 tumors. All $\mathrm{H}$-scores are presented in Table 1 . It was noted that SLC1A5 expression was higher in peripheral than intratumoral areas in MAS98.06 tumors, whereas MAS98.12 tumors were strongly stained throughout the tumor. A similar pattern was found for GLUD1, where MAS98.06 tumors displayed strong staining in peripheral areas and MAS98.12 tumors also displayed strong staining in intratumoral areas close to stromal cells.

\section{Untreated MAS98.06 and MAS98.12 tumors utilize $\left[5^{-13} \mathrm{C}\right]$ glutamine differently}

To determine the consumption and metabolic fate of glutamine in untreated xenografts, we performed in vivo glutamine tracing experiments (experiment 3). Main findings are shown in Fig. 4. Comparing the glutamine-enriched samples with natural abundance spectra (from experiment 1), we found that the xenografts take up significant amounts of glutamine. Both models also use glutamine for anaplerotic fueling of the TCA cycle. However, while MAS98.12 tumors seem to rapidly metabolize glutamine into glutamate, lactate, and alanine, MAS98.06 tumors accumulate significant amounts of glutamine in the tumor tissue. Furthermore, MAS98.06 tumors use glutamine for production of proline, alanine, and lactate, in addition to glutamate and glutamine after one turn in the TCA cycle $\left(\left[1-{ }^{13} \mathrm{C}\right]\right.$ Glu and $\left.\left[1-{ }^{13} \mathrm{C}\right] \mathrm{Gln}\right)$. A detailed presentation of ${ }^{13} \mathrm{C} \mathrm{NMR}$ spectra, in addition to ${ }^{13} \mathrm{C}$ enriched and ${ }^{1} \mathrm{H}$ metabolites for each group, are shown in Additional file 4. Of note, the tumor sample from one mouse with MAS98.12 xenograft had a significantly outlying ${ }^{13} \mathrm{C}$ NMR spectrum, characterized with significantly higher amounts of $\left[5^{-13} \mathrm{C}\right]$ Gln compared to the other samples in the same experimental group (Dixon's $Q$ test for outliers performed on the amount of $\left[5-{ }^{13} \mathrm{C}\right]-\mathrm{Glu}$ in untreated MAS98.12 tumors showed $Q_{\exp }=0.80>$ 


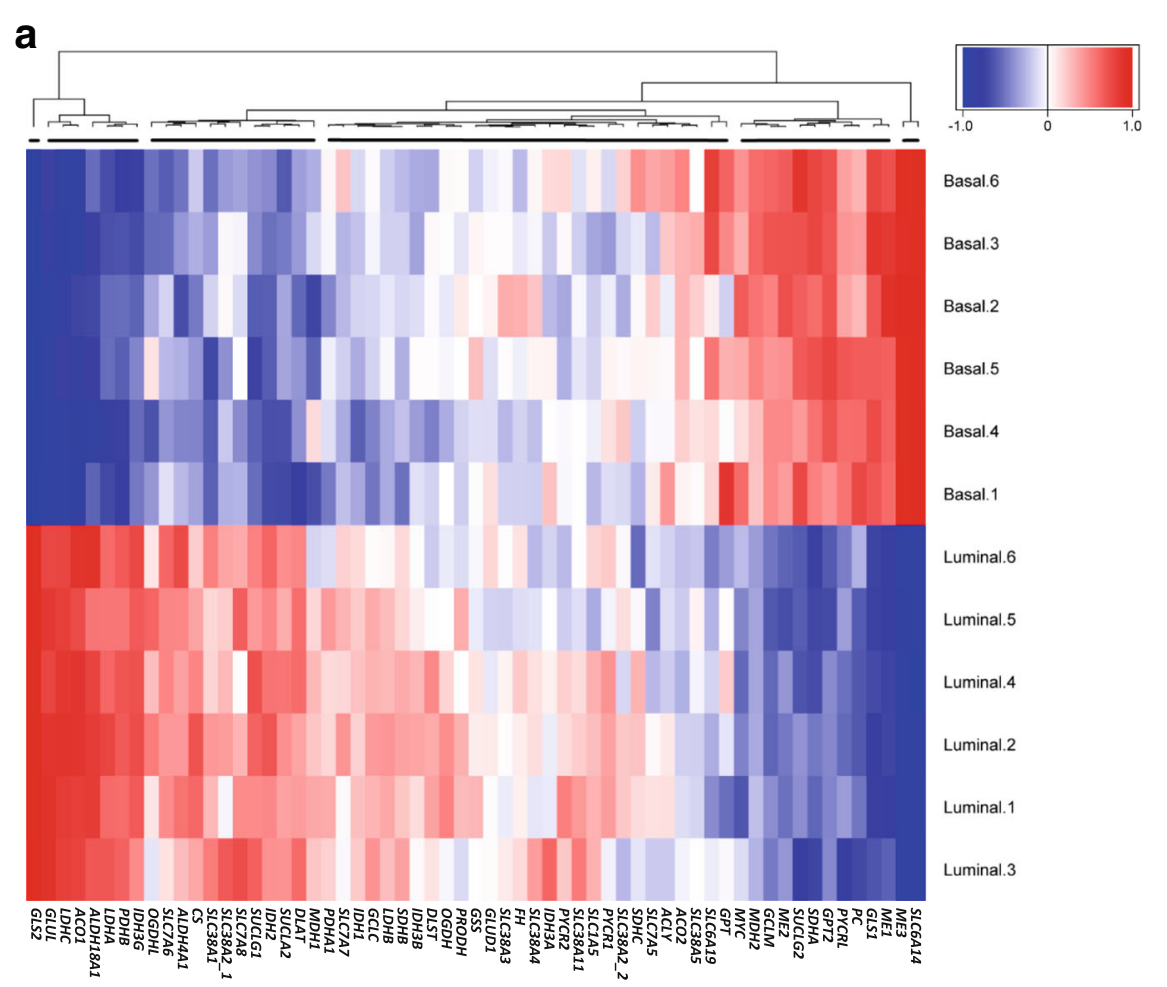

b

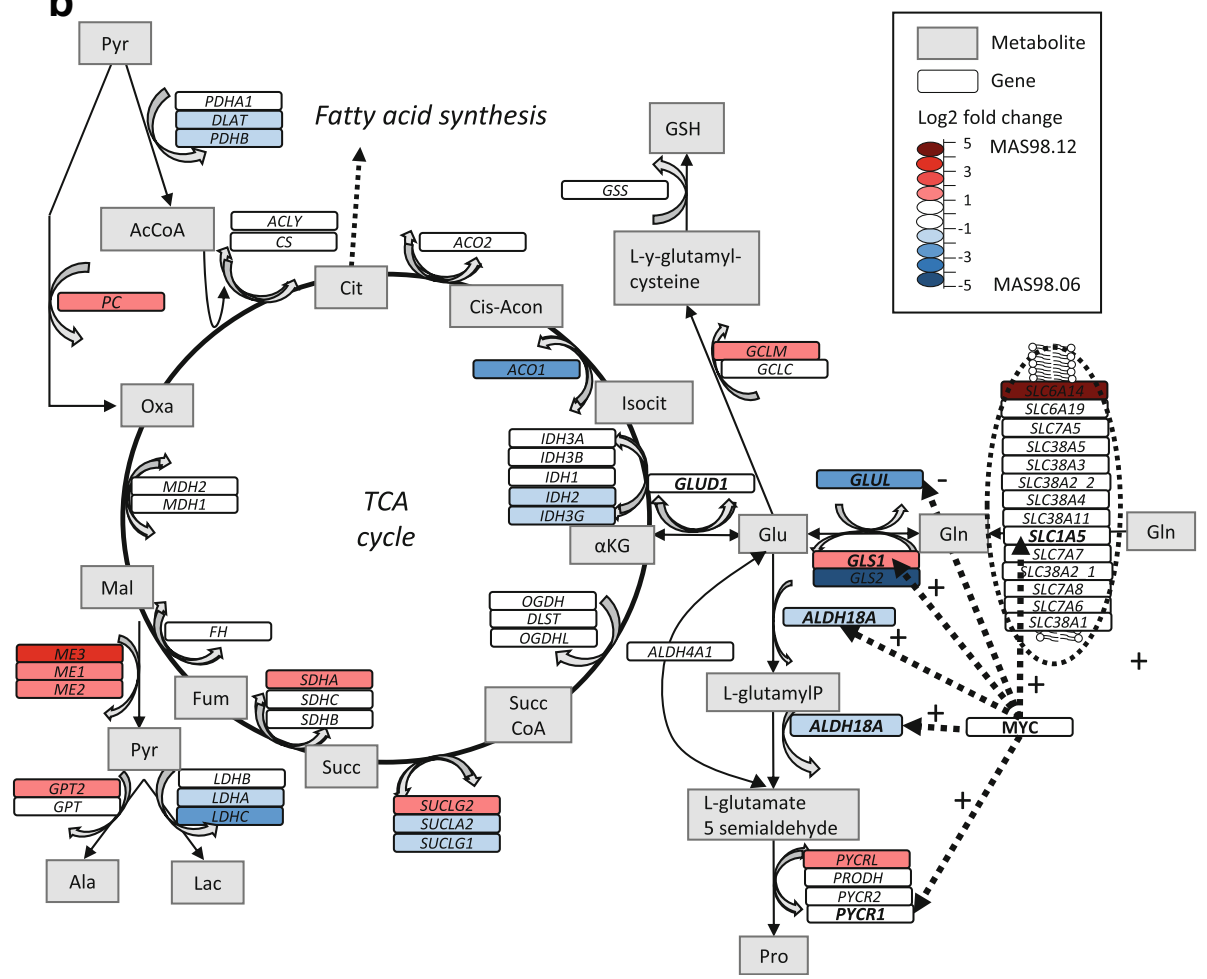

Fig. 2 (See legend on next page.) 
(See figure on previous page.)

Fig. 2 Expression of genes involved in glutaminolysis from untreated MAS98.06 and MAS98.12 tumors. a Heatmap of expression of the 60 selected genes involved in glutaminolysis in untreated MAS98.06 and MAS98.12 tumors. b Differences in gene expression between untreated MAS98.06 and MAS98.12 xenografts. Blue, the gene is significantly higher expressed with at least a two-fold higher expression in MAS98.06; red, the gene is significantly higher expressed with at least two-fold higher expression in MAS98.12; white, the gene is not significantly different expressed between the models. $\log _{2}$-fold change $\left(\log _{2} \mathrm{FC}\right)$ between the two models is indicated by color intensity. Dotted arrows from Myc indicate some of the most important genes that are positively (+) and negatively (-) regulated by Myc, as shown by others [4, 26, 34-36]. Full gene names, enzyme commission numbers (EC numbers), gene expression levels, $q$ values, $\log _{2}$ fold change, and fold changes are specified in Additional file 5

$\left.Q_{\text {critCL95\% }}=0.71\right)[42,43]$. The samples from this mouse were removed from the results.

\section{CB-839 causes depletion of Pro, Ala, and Glu in the MAS98.06 model}

To identify the effect of CB-839 treatment on MAS98.06 and MAS98.12 tumors, we also analyzed ${ }^{13} \mathrm{C}$ NMR spectra from CB-839-treated tumors following infusion with ${ }^{13} \mathrm{C}$-labeled glutamine $\left(\left[5_{-}{ }^{13} \mathrm{C}\right] \mathrm{Gln}\right)$. Main findings from the ${ }^{13} \mathrm{C}$ NMR data from treated models are shown in Fig. 5 . Figure 5 a shows mean ${ }^{13} \mathrm{C}$ NMR spectra along with amounts of selected ${ }^{13} \mathrm{C}$-enriched metabolites (Fig. $5 \mathrm{~b}$ ) in each experimental group. A schematic overview of the most important effects of CB-839 is shown in Fig. 5c, illustrating how CB-839 caused accumulation of $\left[5^{-13} \mathrm{C}\right] \mathrm{Gln}$ in both tumor models, without affecting the $\left[5-{ }^{13} \mathrm{C}\right] \mathrm{Glu}$ levels. In addition, it shows that CB-839 caused depletion of the downstream metabolites $\left[5^{-13} \mathrm{C}\right]$ Pro and $\left[1-{ }^{13} \mathrm{C}\right] \mathrm{Ala}$
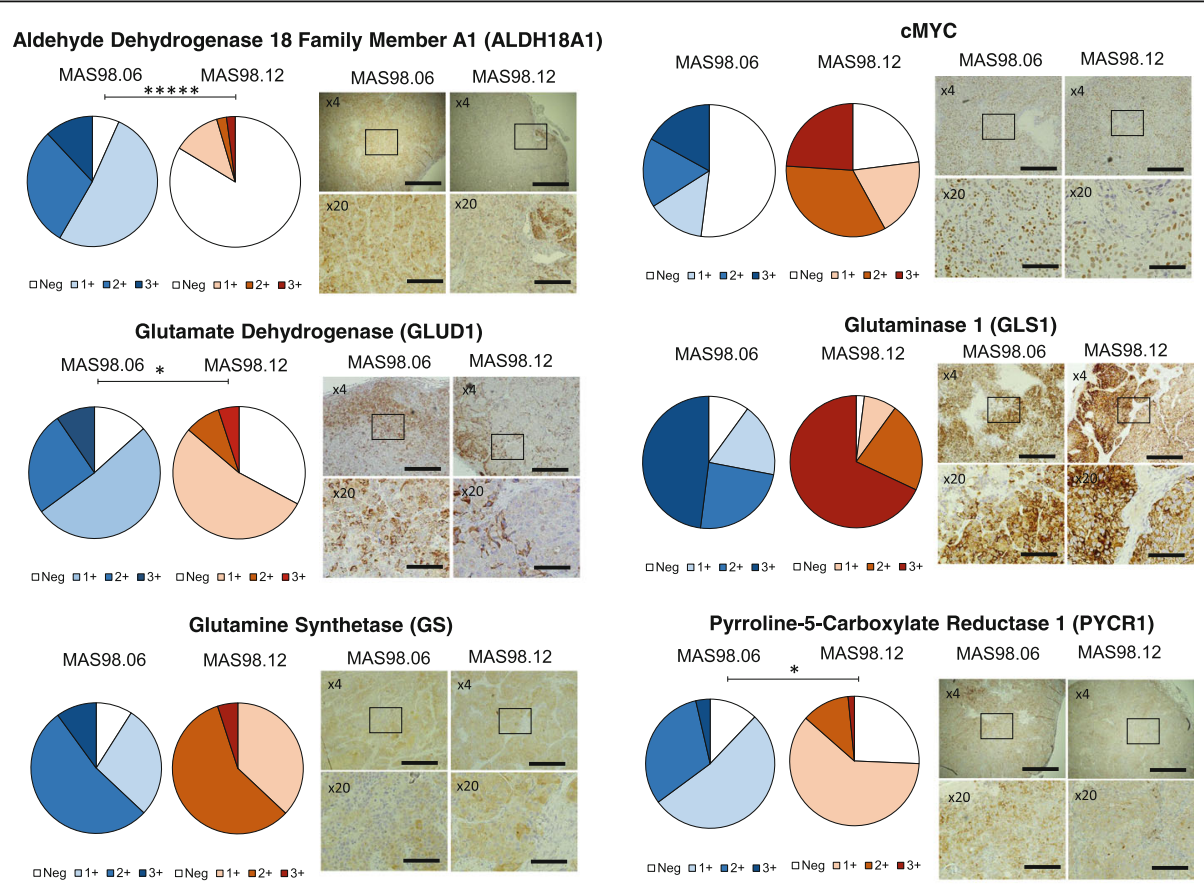

Solute Carrier Family 1 Member 5 (SLC1A5)

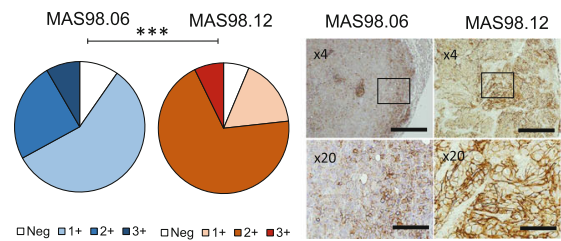

Fig. $3 \mathrm{H}$-scores and representative images from IHC of untreated MAS98.06 and MAS98.12 tumors. The tumors were stained with antibodies against aldehyde dehydrogenase 18 family member A1 (ALDH18A1), CMYC, glutamate dehydrogenase 1 (GLUD1), glutaminase (GLS1), glutamine synthetase (GS), pyrroline-5-carboxylate reductase 1 (PYCR1), and solute carrier family 1 member 5 (SLC1A5). Left columns present the percentage areas with different intensity levels (DAB: 3'-diaminobenzidine) for MAS98.06 (blue shades), and MAS98.12 (red shades) tumors. Neg, no labeling; 1 +, light label; 2+, moderate label; 3+, dark label. Right columns show representative IHC images for each antibody for both MAS98.06 tumors (left) and MAS98.12 tumors (right) in two different magnifications of $\times 4$ and $\times 20$. Scale bar is $200 \mu \mathrm{m}$ for $\times 4$ magnification and $50 \mu \mathrm{m}$ for $\times 20$ magnification. ${ }^{*} p<0.05,{ }^{* * *} p<0.001,{ }^{* * * *} p<0.00001$ 
Table 1 Histo-scores ( $\mathrm{H}$-score, mean \pm stdev) and $p$ values from selected antibodies

\begin{tabular}{llll}
\hline Protein & \multicolumn{2}{l}{ Histo-score } & \multicolumn{2}{l}{\begin{tabular}{l} 
Level of significance \\
\cline { 2 - 3 }
\end{tabular}} & MAS98.06 & MAS98.12 & \\
\hline ALDH18A1 & $147 \pm 25$ & $23 \pm 9$ & $6 \mathrm{e}-06$ \\
CMYC & $99 \pm 52$ & $159 \pm 32$ & ns (0.06) \\
GLUD1 & $131 \pm 35$ & $86 \pm 11$ & 0.03 \\
GLS1 & $210 \pm 25$ & $256 \pm 43$ & ns (0.08) \\
GS & $164 \pm 25$ & $168 \pm 38$ & ns (0.85) \\
PYCR1 & $128 \pm 24$ & $90 \pm 18$ & 0.02 \\
SLC1A5 & $132 \pm 17$ & $178 \pm 6$ & $4 \mathrm{e}-04$ \\
\hline
\end{tabular}

Abbreviations: $A L D H 18 A 1$ aldehyde dehydrogenase 18 family member $A 1$, GLUD1 glutamate dehydrogenase 1, GLS1 glutaminase, GS glutamine synthetase, ns not significant, PYCR1 pyrroline-5-carboxylate reductase 1 , and SLC1A5 solute carrier family 1 member 5 (glutamine transporter)

in MAS98.06 tumors. Box plots of the amount of all ${ }^{13} \mathrm{C}$-enriched metabolites and relevant ${ }^{1} \mathrm{H}$ metabolites for all experimental groups are shown in Additional file 6.

\section{Discussion}

In this study, we have evaluated the treatment response to the glutaminase inhibitor CB-839 in two breast cancer PDX models. Previous studies suggest that basal-like and triple-negative breast cancers are more sensitive to glutaminase inhibitors than luminal-like and ER+ subgroups $[14,16]$. Our findings complement this paradigm; The MAS98.06 xenografts, previously found to belong to the luminal B subtype, were sensitive to glutaminase inhibition whereas the basal-like MAS98.12 xenografts did not respond to treatment.

Previous reports have suggested that TNBC and basal-like breast cancer are particularly dependent on glutamine for proliferation and tumor growth $[14,16$, 44 . Gross and colleagues showed that the TNBC subtype displayed the greatest sensitivity to CB-839 treatment in vitro and that the sensitivity was positively correlated to dependence on extracellular glutamine for growth, high baseline ratio of intracellular glutamate to glutamine (Glu:Gln), and expression of glutaminase (GLS1) [14]. A high Glu:Gln ratio has been proposed as an independent diagnostic entity. Although other studies indicate significant differences in Glu:Gln between breast cancer subgroups, a high Glu:Gln phenotype is observed across all the molecular subtypes [45]. In our experiments, we observed that the triple-negative/basal-like MAS98.12 model had significantly higher gene expression of GLS1 and a trend of higher GLS1 protein

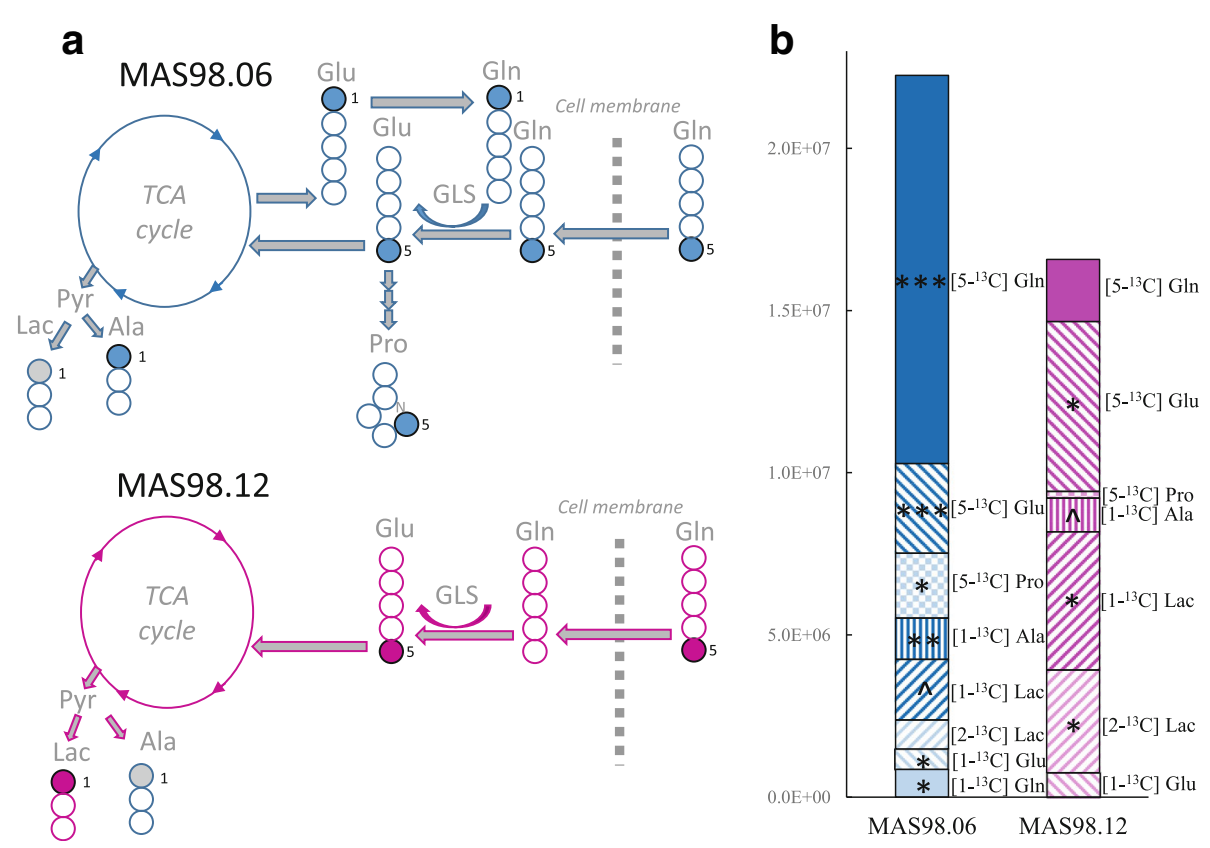

Fig. 4 Utilization of $\left[5^{-13} \mathrm{C}\right] \mathrm{Gln}$ in untreated luminal-like (MAS98.06) and basal-like (MAS98.12) tumor xenografts. a ${ }^{13} \mathrm{C}$ labeling patterns in MAS98.06 and MAS98.12 tumors after administration of $\left[5^{-13} \mathrm{C}\right]$ Gln. MAS98.06 tumors (blue) use $\left[5-{ }^{13} \mathrm{C}\right]$ Gln to produce $\left[5_{-}{ }^{13} \mathrm{C}\right] \mathrm{Glu},\left[5^{-13} \mathrm{C}\right]$ Pro, $\left[1-{ }^{13} \mathrm{C}\right]$ Ala, $\left[1-{ }^{13} \mathrm{C}\right] \mathrm{Glu}$, and $\left[1-{ }^{13} \mathrm{C}\right]$ Lac (gray, only borderline significant). They also store a significant amount of $\left[5-{ }^{13} \mathrm{C}\right]$ Gln in the tumors. MAS98.12 tumors (pink) take up $\left[5-{ }^{13} \mathrm{C}\right] \mathrm{Gln}$ and use it for production of $\left[5-{ }^{13} \mathrm{C}\right] \mathrm{Glu},\left[1-{ }^{13} \mathrm{C}\right] \mathrm{LaC}$ and $\left[5-{ }^{13} \mathrm{C}\right]$ Ala (gray, only borderline significant). $\mathbf{b}$ Amount of ${ }^{13} \mathrm{C}$-labeled metabolites in the tumors, calculated by subtracting natural abundance spectra from ${ }^{13} \mathrm{C}$-enriched spectra. Stars $\left({ }^{*}\right)$ indicate that there is a significantly higher amount of the metabolite in ${ }^{13} \mathrm{C}$-enriched samples compared to natural abundance samples, while up arrowheads $(\wedge)$ indicate borderline significance. The total amount of ${ }^{13} \mathrm{C}$-labeled metabolites were not significantly different between the two models. $\wedge p<0.1,{ }^{*} p<0.05,{ }^{* *} p<0.01,{ }^{* * *} p<0.001$. Abbreviations: Ala, alanine; Gln, glutamine; GLS1, glutaminase; Glu, glutamate; Lac, lactate; Pro, proline; Pyr, pyruvate; TCA, tricarboxylic acid 


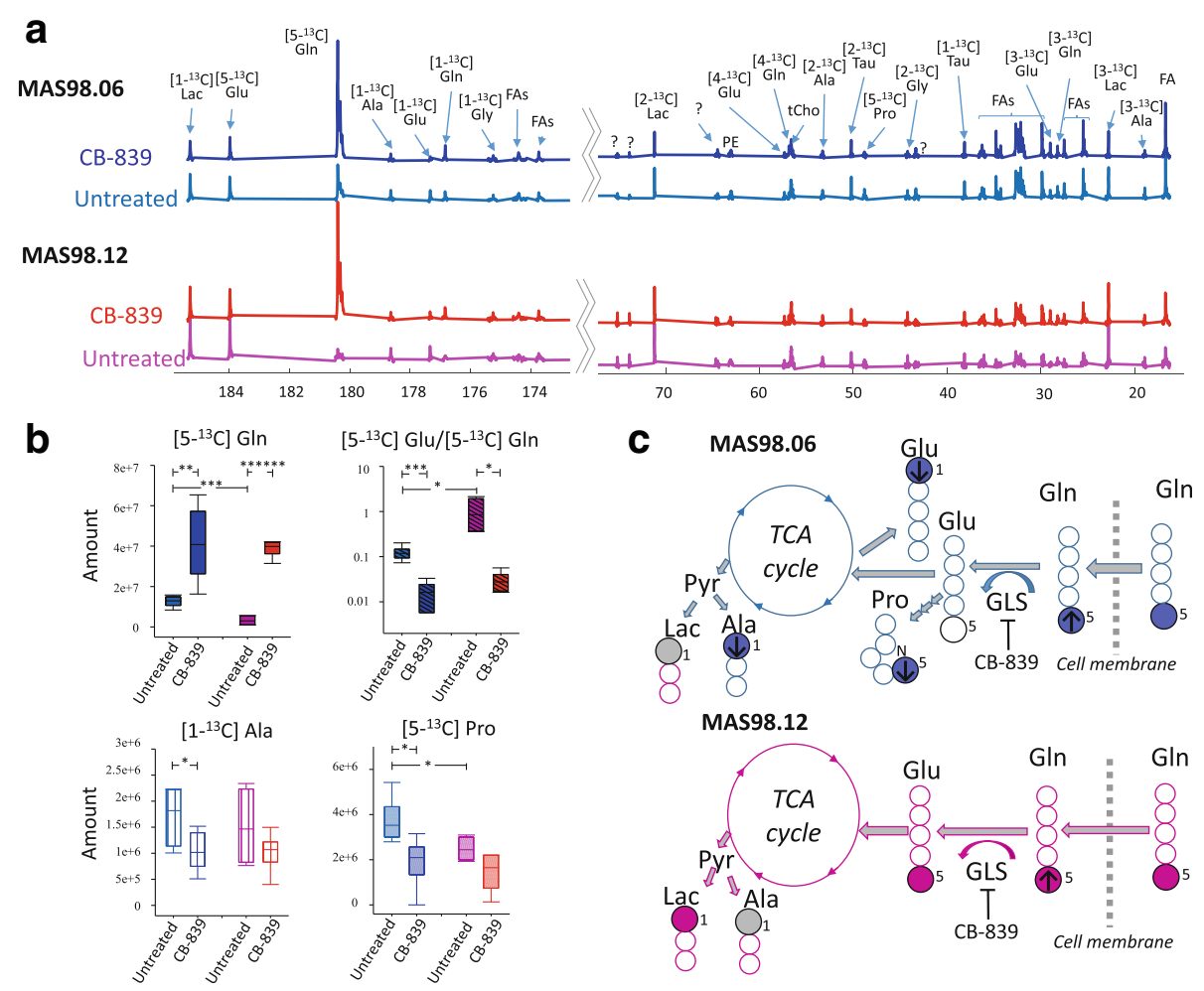

Fig. 5 Effect of CB-839 on glutamine metabolism in MAS98.06 and MAS98.12 PDX tumors. a Average ${ }^{13} \mathrm{C}$ NMR spectra (173.5-185.5 ppm and 7513 ppm) for CB-839-treated and untreated MAS98.06 (blue) and MAS98.12 (red) tumors receiving ${ }^{13} \mathrm{C}$-labeled glutamine. The contribution to the average from each individual NMR spectrum is scaled to the sample mass. b Quantified amounts of selected ${ }^{13} \mathrm{C}-$ labeled metabolites in each experimental group: ${ }^{13} \mathrm{C}$ glutamine $\left(\left[5-{ }^{13} \mathrm{C}\right] \mathrm{Gln}\right)$, glutamate to glutamine ratio $\left(\left[5-{ }^{13} \mathrm{C}\right] \mathrm{Glu} /\left[5^{-13} \mathrm{C}\right] \mathrm{Gln}\right)$, alanine $\left(\left[1-{ }^{13} \mathrm{C}\right]\right.$ Ala), and proline $\left(\left[5-{ }^{13} \mathrm{C}\right]\right.$ Pro) in the experimental groups. c MAS98.06 tumors take up and store glutamine (GIn) and use glutamine to produce glutamate (Glu), proline (Pro), alanine (Ala), lactate (Lac), and glutamate (Glu) through one turn in TCA cycle, as indicated by the filled blue circles (gray circle for Lac, only borderline significant). CB-839 causes an accumulation of Gln (arrow up) and reduced amounts of Pro, Al, and Glu (arrows down) in the tumors (only $\left[1-{ }^{13} \mathrm{C}\right]$ Glu, which is created after one turn in TCA cycle, is reduced). MAS98.12 tumors use glutamine (GIn) to produce Glu, Lac, and Ala as indicated by filled pink circles (gray circle for Ala, only borderline significant). CB-839 causes accumulation of Gln in MAS98.12 tumors, but does not significantly change the amount of any other ${ }^{13} \mathrm{C}$-enriched metabolites ${ }^{*} p<0.05,{ }^{* *} p<0.01,{ }^{* * *} p<0.001,{ }^{* * *} p<0.0001$

expression, and a significantly higher Glu:Gln ratio compared to the luminal B/ER+ MAS98.06 models. Despite the accordance with previously proposed predictive biomarkers, the MAS98.12 model did not respond to CB-839 treatment, suggesting that response and resistance depend on other mechanisms regulating glutamine metabolism. Metabolic redundancy or plasticity can rescue cancer cells from glutaminase inhibition, and our understanding of metabolic characteristics within the molecular breast cancer subtypes remains limited. Several studies have demonstrated metabolic variability within subtypes and a lack of correlation between metabolic and transcriptomic traits [46, 47]. Although the triple-negative phenotype has been suggested to be particularly dependent on glutaminolysis, it has been shown that as many as $25 \%$ of these do not express GLS1 as determined by immunohistochemistry [48]. In a panel of 26 PDX models, we found more than ten-fold difference in the expression of GLS1 within the basal-like subtype
(Additional file 2). Similar variability was observed for SLC1A5, GS, and GLUD1. Both MAS98.06 and MAS98.12 displayed GLS1 expression higher than the average for the cohort. No clear distinctions between basal-like and luminal B xenografts were observed for any of the genes. This indicates that although the two subtypes display metabolic differences on the population level, it must be expected that individuals within each subtype display atypical metabolic characteristics.

In order to better understand mechanisms responsible for response and resistance to glutaminase inhibitors, we studied glutamine utilization in the two xenografts by infusion of ${ }^{13} \mathrm{C}$-enriched glutamine and ex vivo ${ }^{13} \mathrm{C}$ NMR tumor analysis, combined with gene- and protein expression data. From the ${ }^{13} \mathrm{C}$ HR MAS MRS analysis on untreated models, we saw that the two xenograft models utilize glutamine differently. Although the total glutamine consumption was similar in the two models, the main metabolic fate of glutamine in the non-responding 
MAS98.12 was conversion into glutamate, lactate, and alanine. In contrast, the responding MAS98.06 tumors store significant amounts of glutamine in the tumors, but also use glutamine for synthesis of proline, alanine, and lactate. Both models use a similar fraction of the glutamine to feed the TCA cycle, as seen by the presence of $\left[1-{ }^{13} \mathrm{C}\right]$ glutamate (MAS98.06) and $\left[1-{ }^{13} \mathrm{C}\right]$ lactate (MAS98.12).

A possible scenario that may explain why some tumors are insensitive to CB-839 is that they use glycolysis instead of glutaminolysis for anaplerotic feeding of the TCA cycle. Pyruvate can enter the TCA cycle through dehydrogenation of pyruvate into acetyl-CoA (which is catalyzed by pyruvate dehydrogenase (PDH)) or through carboxylation of pyruvate into oxaloacetate (catalyzed by pyruvate carboxylase (PC)). However, we have previously found that following $\left[1-{ }^{13} \mathrm{C}\right]$ Glc injection, MAS98.12 tumors feed less glucose into the TCA cycle than MAS98.06 tumors, both via PDH and PC [49]. Differences in anaplerotic fueling of the TCA by glucose can therefore not explain the differences in response to CB-839 observed in this study.

It is well established that the signaling landscape plays critical roles in regulating proliferation and tumor growth [50]. The transcription factor Myc affects glutamine metabolism by enhancing glutamine uptake, glutaminase activity, and upregulation of proline metabolism [4, 26, 34-36]. Myc may trigger addiction to glutamine, which is observed both in vitro and in vivo $[8,50]$. Several transporters are capable of transporting glutamine across the plasma membrane [26]. Among these, SLC1A5 has received increased attention because its expression is upregulated in many cancer types, including triple-negative breast cancer [27]. We observed that MAS98.12 xenografts display a borderline significant higher expression of MYC than MAS98.06 xenografts. This may explain the higher expression of the glutamine transporter SLC1A5 protein and GLS1 mRNA in MAS98.12. However, expression of MYC was not associated with increased glutamine consumption or proline synthesis in our models.

In tumor tissue from the responding MAS98.06 model, we saw a depletion of proline and alanine after treatment. Alanine is produced from pyruvate, which can be synthesized using several routes, including glycolysis. Proline, on the other hand, is a conditionally essential amino acid [51]. In untreated MAS98.06 xenografts, a significant fraction of the injected $\left[5{ }^{-13} \mathrm{C}\right]$ glutamine was converted to $\left[5-{ }^{13} \mathrm{C}\right]$ proline. This is consistent with gene and protein expression data, showing that ALDH18A1 and PYCR1 were more highly expressed in MAS98.06 than MAS98.12 both on gene and protein expression level. These results indicate that MAS98.06 tumors have a higher flux from glutamate to proline compared to MAS98.12 tumors. Recent research by Tang and colleagues has identified proline as an important metabolite in cancer, linked to adaption to tumor hypoxia [6]. They showed that hypoxic microenvironments activated proline metabolism via upregulation of $A L D H 18 A 1$ in tumor samples from patients with hepatocellular carcinoma. We have previously shown that the luminal-like MAS98.06 tumors are more hypoxic than basal-like MAS98.12 tumors [20]. It could therefore be speculated that MAS98.06 tumors adapt to hypoxic microenvironments through activation of proline-mediated mechanisms. Depletion of proline following CB-839 treatment to the MAS98.06 models could leave the tumors unable to handle hypoxic stress, consequently inhibiting the growth of these tumors.

Overall, our results indicate that current biomarkers suggested for predicting response to glutaminase inhibition do not fully capture the complexity of glutamine metabolism in cancer and that the response to glutaminase inhibitors depends on the individual tumor's ability to compensate for reduced glutamate availability. One possible explanation for the glutamine dependence in MAS98.06 tumors is that they use glutamine in proline biosynthesis, for adaption to hypoxic microenvironments.

\section{Conclusion}

This work demonstrates that the glutaminase inhibitor CB-839, which currently is in early clinical trials for TNBC patients, significantly inhibits tumor growth in one of the breast cancer PDX models, the luminal b/ER + MAS98.06 model. However, it had no effect on tumor growth in the other basal-like/triple-negative MAS98.12 PDX model. Gene expression analysis and IHC show that the responding model (MAS98.06) has higher gene and protein expressions of enzymes involved in the conversion of glutamine to proline. ${ }^{13} \mathrm{C}$ NMR data of tissue from untreated tumors supports these findings, as the responding model has higher proline synthesis than the non-responding model. Following treatment with CB-839, ${ }^{13} \mathrm{C}$ NMR demonstrated proline depletion in the responding model. One possible explanation is that the responding MAS98.06 model, which is found to be more hypoxic than non-responding MAS98.12, is dependent on proline synthesis from glutamine to adapt to the hypoxic microenvironment. Our study illustrates the shortcomings of currently proposed predictive biomarkers of response to glutaminase inhibition in breast cancer.

\section{Additional files}

Additional file 1: Figure with an overview of the PDX models and experimental design of the study. Abbreviations: HR MAS MRS, high- 
resolution magic angle spinning MR spectroscopy: IHC, immunohistochemistry; N.A., natural abundance (PPTX 359 kb)

Additional file 2: Figure with gene expression of SLC1A5, GLS1, GLUL, and GLUD1 in 19 basal-like and 7 luminal B PDX models. The gene expression data of SLC1A5, GLS1, GLUL, and GLUD1 in 19 basal-like PDX tumors (red) and 7 basal-like PDX tumors (cyan) is presented in waterfall plots. The expressions for each genes are mean normalized. The microarray data are collected from the Gene Expression Omnibus (GEO) with accession number GSE44666 (PPTX 31009 kb)

Additional file 3: Table with overview of primary antibodies. Gives information about species reactivity, suppliers and product information, host species, and antibody dilution used during immunohistochemistry (IHC) staining (DOCX $13 \mathrm{~kb}$ )

Additional file 4: Figure showing HR MAS MRS data from untreated MAS98.06 and MAS98.12 tumors. a) Average ${ }^{13} \mathrm{C}$ HR MAS MRS spectra calculated by subtracting natural abundance spectra from ${ }^{13} \mathrm{C}$ enriched spectra. Positive signals with stars $\left(^{*}\right)$ indicate that there is a significantly higher amount of the metabolite in ${ }^{13} \mathrm{C}$-enriched samples compared to natural abundance samples, whereas up arrowheads $(\wedge)$ indicate borderline significance. b) Amount of ${ }^{13} \mathrm{C}$-labeled metabolites in the tumors, calculated by subtracting natural abundance spectra from ${ }^{13} \mathrm{C}$-enriched spectra. Stars $\left(^{*}\right)$ indicate that there is a significantly higher amount of the metabolite in ${ }^{13} \mathrm{C}-$ enriched samples compared to natural abundance samples, and up arrowheads $(\wedge)$ indicate borderline significance. The total amount of ${ }^{13} \mathrm{C}$ labeled metabolites were not significantly different between the two models. c) Box plots showing the amount of the ${ }^{13} \mathrm{C}$-labeled metabolites subtracted with the amount of the metabolites from the natural abundance spectra. d) Amounts of selected metabolites from ${ }^{1} \mathrm{H}$ spectra calculated from natural abundance and ${ }^{13} \mathrm{C}$-enriched samples. ${ }^{*} p<0.05,{ }^{* *} p<0.01,{ }^{* * *} p<0.001$. Abbreviations: Ala, alanine; Gln, glutamine; GLS; glutaminase; Glu, glutamate; Lac, lactate; Pro, proline; Pyr, pyruvate; TCA, tricarboxylic acid (PPTX 1939 kb)

Additional file 5: Table with gene expression data from the 60 selected genes. The table gives information about gene symbols, probe names, the associated EC number, $p$ values and $q$ values, gene expression levels for both models ( $\log _{2}$ transformed), $\log _{2}$ fold change, and fold change. The table includes the same color coding system as Fig. 2 in the article. The seven selected key genes are marked in bold (DOCX $23 \mathrm{~kb}$ )

Additional file 6: Figure showing the effect of CB-839 in MAS98.06 and MAS98.12 tumors. a) Average ${ }^{13} \mathrm{C}$ NMR spectra (173.5-185.5 ppm and 7513 ppm) for CB-839-treated and untreated MAS98.06 and MAS98.12 models receiving ${ }^{13} \mathrm{C}$-labeled glutamine. b) Quantified amounts of ${ }^{13} \mathrm{C}$-labeled metabolites in each experimental group: ${ }^{13} \mathrm{C}$ glutamine $\left(\left[5-{ }^{13} \mathrm{C}\right]\right.$ Gln), glutamate $\left(\left[5-{ }^{13} \mathrm{C}\right] \mathrm{Glu}\right.$ and $\left.\left[1-{ }^{13} \mathrm{C}\right] \mathrm{Glu}\right)$, alanine $\left(\left[1-{ }^{13} \mathrm{C}\right] \mathrm{Ala}\right)$, lactate $\left(\left[1-{ }^{13} \mathrm{C}\right] \mathrm{Lac}\right.$, proline $\left(\left[5^{13} \mathrm{C}\right]\right.$ Pro), and glutamate to glutamine ratio $\left(\left[5-{ }^{-13} \mathrm{C}\right] \mathrm{Glu} /\left[5^{-13} \mathrm{C}\right] \mathrm{Gln}\right)$ in the experimental groups. C) MAS98.06 tumors take up and store glutamine (Gln) in the tumors and use glutamine to produce proline (Pro), alanine (Ala), lactate (Lac), and glutamate (Glu) through one turn in TCA cycle as indicated by filled blue circles (Lac only borderline significant, gray circle). CB-839 causes an accumulation of Gln (arrow up) and reduced amounts of Pro, Ala, and Glu (arrows down) in the tumors (only $\left[1-{ }^{13} \mathrm{C}\right]$ Glu, which is created after one turn in TCA cycle, is reduced). MAS98.12 tumors use glutamine (GIn) to produce Glu, Lac, and Ala as indicated by filled pink circles (Ala only borderline significant, gray circle). (B-839 causes accumulation of GIn in MAS98.12 tumors, but does not significantly change the amount of any other ${ }^{13} \mathrm{C}$-enriched metabolites. d) Quantified amount of relevant metabolites from ${ }^{1} \mathrm{H}$ spectra. ${ }^{*} p<0.05,{ }^{* *} p<0.01,{ }^{* * *} p<0.001,{ }^{* * *} p<0.0001$ (PPTX $339 \mathrm{~kb}$ )

\section{Abbreviations}

Ala: Alanine; ALDH18A1: Aldehyde dehydrogenase 18 family member A1; DAB: 3,3'-Diaminobenzidine; ER+: Estrogen receptor positive; GIn: Glutamine; GLS1/GLS2: Glutaminase 1/glutaminase 2; Glu: Glutamate; GLUD1: Glutamate dehydrogenase 1; GLUL: Glutamine synthetase (gene); GS: Glutamine synthetase (protein); HR MAS MRS: High-resolution magic angle spinning MR spectroscopy; HRP: Horseradish peroxidase; Lac: Lactate; NMR: Nuclear magnetic resonance; PDH: Pyruvate dehydrogenase; PDX: Patient-derived xenograft; Pro: Proline; PYCR1: Pyrroline-5-carboxylate reductase 1; SLC1A5: Solute carrier family 1 member 5; SLC38A2: Solute carrier family 38 member 2; TNBC: Triple-negative breast cancer

\section{Acknowledgements}

The authors want to thank the following departments and companies: Dept. of Tumor Biology, Oslo University Hospital, for providing xenograft-bearing mice; Comparative Medicine Core Facility (CoMed), Norwegian University of Science and Technology (NTNU), for facilitating with animal experiments; Calithere Biosciences for kindly donating CB-839; MR Core Facility, NTNU, for facilitating with HR MAS MRS; and Cellular \& Molecular Imaging Core Facility (CMIC), NTNU and Covance Inc., for performing immunohistological stainings and scoring.

\section{Funding}

This study was supported by grants from the Research Council of Norway and a research fellowship to $\mathrm{BH}$ through the Marie Sklodowska-Curie Actions/Scientia Fellowship programme at the University of Oslo.

\section{Availability of data and materials}

Data generated during this study are included in this published article and its additional files. Gene expression datasets analyzed during the current study are available in the Gene Expression Omnibus (GEO) repository, https:// www.ncbi.nlm.nih.gov/geo/query/acc.cgi?acc=GSE37543, https://www.ncbi. nlm.nih.gov/geo/query/acc.cgi?acc=GSE44666.

\section{Authors' contributions}

MTG was responsible for the animal ${ }^{13} \mathrm{C}$ tracing experiments, acquired and analyzed HR MAS MRS data, scored IHC section data, and drafted the manuscript. $\mathrm{BH}$ was responsible for the analyses of the gene expression data and was involved in the drafting of the manuscript. HMT and JK were involved in the animal experiments. IMH acquired and analyzed HR MAS MRS data. MHH, HMT, and MBR analyzed gene expression data. GMM coordinated the animal models. SAM designed and coordinated the study, scored the IHC sections, and was involved in the drafting of the manuscript. All authors critically read, commented, and approved the final manuscript.

\section{Ethics approval and consent to participate}

All procedures and experiments involving animals were approved by the Norwegian Animal Research Authority (FOTS ID: 7713 and 9126) and carried out according to the European Convention for the Protection of Vertebrates used for Scientific Purposes.

\section{Consent for publication}

Not applicable.

\section{Competing interests}

The authors declare that they have no competing interests.

\section{Publisher's Note}

Springer Nature remains neutral with regard to jurisdictional claims in published maps and institutional affiliations.

\section{Author details}

'Department of Circulation and Medical Imaging, Norwegian University of Science and Technology (NTNU), 7489 Trondheim, Norway. ${ }^{2}$ Department of Tumor Biology, Institute for Cancer Research, Oslo University Hospital, Oslo, Norway. ${ }^{3}$ Faculty of Medicine, Institute of Clinical Medicine, University of Oslo, Oslo, Norway. ${ }^{4}$ Department of Physics, NTNU, Trondheim, Norway. ${ }^{5}$ Department of Radiology and Nuclear Medicine, St. Olavs Hospital, Trondheim University Hospital, Trondheim, Norway. ${ }^{6}$ Department of Cancer Research and Molecular Medicine, NTNU, Trondheim, Norway. ${ }^{7}$ Clinic of Surgery, St. Olav's Hospital, Trondheim University Hospital, Trondheim, Norway. ${ }^{8}$ Institute of Medical Biology, Faculty of Health Sciences, University of Troms $\varnothing$ - The Arctic University of Norway, Troms $\varnothing$, Norway. ${ }^{9}$ Department of Clinical and Molecular Medicine, NTNU, Trondheim, Norway. ${ }^{10}$ Department of Pharmacy, Nord Universitet, Namsos, Norway.

Received: 4 January 2019 Accepted: 16 April 2019

Published online: 14 May 2019

\section{References}

1. Hanahan D, Weinberg RA. Hallmarks of cancer: the next generation. Cell. 2011;144(5):646-74.

2. Hanahan D, Weinberg RA. The hallmarks of cancer. Cell. 2000;100(1):57-70. 
3. Balendiran GK, Dabur R, Fraser D. The role of glutathione in cancer. Cell Biochem Funct. 2004;22(6):343-52.

4. Phang JM, Liu W, Hancock CN, Fischer JW. Proline metabolism and cancer: emerging links to glutamine and collagen. Curr Opin Clin Nutr Metab Care. 2015;18(1):71-7.

5. Liu W, Le A, Hancock C, Lane AN, Dang CV, Fan TW, et al. Reprogramming of proline and glutamine metabolism contributes to the proliferative and metabolic responses regulated by oncogenic transcription factor c-MYC. Proc Natl Acad Sci U S A. 2012;109(23):8983-8.

6. Tang L, Zeng J, Geng P, Fang C, Wang Y, Sun M, et al. Global metabolic profiling identifies a pivotal role of proline and hydroxyproline metabolism in supporting hypoxic response in hepatocellular carcinoma. Clin Cancer Res. 2018;24(2):474-85

7. Yuneva M, Zamboni N, Oefner P, Sachidanandam R, Lazebnik Y. Deficiency in glutamine but not glucose induces MYC-dependent apoptosis in human cells. J Cell Biol. J Cell Biol. 2007;178:93-105.

8. Wise DR, DeBerardinis RJ, Mancuso A, Sayed N, Zhang XY, Pfeiffer HK, et al. Myc regulates a transcriptional program that stimulates mitochondrial glutaminolysis and leads to glutamine addiction. Proc Natl Acad Sci U S A. 2008;105(48):18782-7.

9. Huang F, Zhang Q, Ma H, Lv Q, Zhang T. Expression of glutaminase is upregulated in colorectal cancer and of clinical significance. Int J Clin Exp Pathol. 2014;7(3):1093-100.

10. Seltzer MJ, Bennett BD, Joshi AD, Gao P, Thomas AG, Ferraris DV, et al. Inhibition of glutaminase preferentially slows growth of glioma cells with mutant IDH1. Cancer Res. 2010;70(22):8981-7.

11. Son J, Lyssiotis CA, Ying H, Wang X, Hua S, Ligorio M, et al. Glutamine supports pancreatic cancer growth through a KRAS-regulated metabolic pathway. Nature. 2013;496(7443):101-5.

12. Filipp FV, Ratnikov B, De Ingeniis J, Smith JW, Osterman AL, Scott DA. Glutamine-fueled mitochondrial metabolism is decoupled from glycolysis in melanoma. Pigment Cell Melanoma Res. 2012;25(6):732-9.

13. Wang J-B, Erickson JW, Fuji R, Ramachandran S, Gao P, Dinavahi R, et al. Targeting mitochondrial glutaminase activity inhibits oncogenic transformation. Cancer Cell. 2010;18(3):207-19.

14. Gross MI, Demo SD, Dennison JB, Chen L, Chernov-Rogan T, Goyal B, et al. Antitumor activity of the glutaminase inhibitor CB-839 in triple-negative breast cancer. Mol Cancer Ther. 2014;13(4):890-901.

15. Krebs HA. Metabolism of amino-acids: the synthesis of glutamine from glutamic acid and ammonia, and the enzymic hydrolysis of glutamine in animal tissues. Biochem J. 1935;29(8):1951-69.

16. Lampa M, Arlt H, He T, Ospina B, Reeves J, Zhang B, et al. Glutaminase is essential for the growth of triple-negative breast cancer cells with a deregulated glutamine metabolism pathway and its suppression synergizes with mTOR inhibition. PLoS One. 2017;12(9):e0185092.

17. Harding JJ, Telli ML, Munster PN, Le MH, Molineaux C, Bennett MK, et al. Safety and tolerability of increasing doses of CB-839, a first-in-class, orally administered small molecule inhibitor of glutaminase, in solid tumors. J Clin Oncol. 2015;33(15-suppl):2512.

18. Bergamaschi A, Hjortland GO, Triulzi T, Sorlie T, Johnsen H, Ree AH, et al. Molecular profiling and characterization of luminal-like and basal-like in vivo breast cancer xenograft models. Mol Oncol. 2009;3(5-6):469-82.

19. Grinde MT, Skrbo N, Moestue SA, Rodland EA, Borgan E, Kristian A, et al. Interplay of choline metabolites and genes in patient-derived breast cancer xenografts. Breast Cancer Res. 2014;16(1):R5.

20. Huuse EM, Moestue SA, Lindholm EM, Bathen TF, Nalwoga H, Krüger K, et al. In vivo MRI and histopathological assessment of tumor microenvironment in luminal-like and basal-like breast cancer xenografts. J Magn Reson Imaging. 2012;35(5):1098-107.

21. Borgan E, Lindholm EM, Moestue S, Maelandsmo GM, Lingjaerde OC, Gribbestad IS, et al. Subtype-specific response to bevacizumab is reflected in the metabolome and transcriptome of breast cancer xenografts. Mol Oncol. 2013;7(1):130-42.

22. Smyth GK. Linear models and empirical bayes methods for assessing differentia expression in microarray experiments. Stat Appl Genet Mol Biol. 2004;3:Article3.

23. Kanehisa M, Goto SKEGG. Kyoto encyclopedia of genes and genomes. Nucleic Acids Res. 2000;28(1):27-30.

24. Hirsch FR, Varella-Garcia M, Bunn PA Jr, Di Maria MV, Veve R, Bremmes RM, et al. Epidermal growth factor receptor in non-small-cell lung carcinomas: correlation between gene copy number and protein expression and impact on prognosis. J Clin Oncol. 2003;21(20):3798-807.
25. Lingjaerde OC, Steen CB. Personal communication; 2018.

26. Bhutia YD, Ganapathy V. Glutamine transporters in mammalian cells and their functions in physiology and cancer. Biochim Biophys Acta. 2016; 1863(10):2531-9.

27. van Geldermalsen M, Wang Q, Nagarajah R, Marshall AD, Thoeng A, Gao D, et al. ASCT2/SLC1A5 controls glutamine uptake and tumour growth in triple-negative basal-like breast cancer. Oncogene. 2015;35:3201.

28. Hassanein M, Hoeksema MD, Shiota M, Qian J, Harris BK, Chen H, et al. SLC1A5 mediates glutamine transport required for lung cancer cell growth and survival. Clin Cancer Res. 2013;19(3):560-70.

29. Kung H-N, Marks JR, Chi J-T. Glutamine synthetase is a genetic determinant of cell type-specific glutamine independence in breast epithelia. PLoS Genet. 2011;7(8):e1002229.

30. Timmerman Luika A, Holton T, Yuneva M, Louie Raymond J, Padró M, Daemen A, et al. Glutamine sensitivity analysis identifies the xCT antiporter as a common triple-negative breast tumor therapeutic target. Cancer Cell. 2013:24(4):450-65.

31. Yang C, Sudderth J, Dang T, Bachoo RG, McDonald JG, DeBerardinis RJ. Glioblastoma cells require glutamate dehydrogenase to survive impairments of glucose metabolism or Akt signaling. Cancer Res. 2009;69(20):7986.

32. De Ingeniis J, Ratnikov B, Richardson AD, Scott DA, Aza-Blanc P, De SK, et al. Functional specialization in proline biosynthesis of melanoma. PLoS One. 2012;7(9):e45190.

33. Ding J, Kuo M-L, Su L, Xue L, Luh F, Zhang H, et al. Human mitochondrial pyrroline-5-carboxylate reductase 1 promotes invasiveness and impacts survival in breast cancers. Carcinogenesis. 2017;38(5):519-31.

34. Craze ML, Cheung H, Jewa N, Coimbra NDM, Soria D, El-Ansari R, et al. MYC regulation of glutamine-proline regulatory axis is key in luminal $\mathrm{B}$ breast cancer. Br J Cancer. 2018;118(2):258-65.

35. Gao P, Tchernyshyov I, Chang TC, Lee YS, Kita K, Ochi T, et al. c-Myc suppression of miR-23a/b enhances mitochondrial glutaminase expression and glutamine metabolism. Nature. 2009:458(7239):762-5.

36. Wise DR, Thompson CB. Glutamine addiction: a new therapeutic target in cancer. Trends Biochem Sci. 2010;35(8):427-33.

37. John T, Liu G, Tsao MS. Overview of molecular testing in non-small-cell lung cancer: mutational analysis, gene copy number, protein expression and other biomarkers of EGFR for the prediction of response to tyrosine kinase inhibitors. Oncogene. 2009;28(Suppl 1):S14-23.

38. Choudhury KR, Yagle KJ, Swanson PE, Krohn KA, Rajendran JG. A robust automated measure of average antibody staining in immunohistochemistry images. J Histochem Cytochem. 2010;58(2):95-107.

39. Marin-Valencia I, Yang C, Mashimo T, Cho S, Baek H, Yang XL, et al. Analysis of tumor metabolism reveals mitochondrial glucose oxidation in genetically diverse human glioblastomas in the mouse brain in vivo. Cell Metab. 2012;15(6):827-37.

40. Wider G, Dreier L. Measuring protein concentrations by NMR spectroscopy. J Am Chem Soc. 2006;128(8):2571-6.

41. Eilers PH. Parametric time warping. Anal Chem. 2004;76(2):404-11.

42. Dean RB, Dixon WJ. Simplified statistics for small numbers of observations. Anal Chem. 1951:23(4):636-8.

43. Rorabacher DB. Statistical treatment for rejection of deviant values: critical values of Dixon's "Q" parameter and related subrange ratios at the 95\% confidence level. Anal Chem. 1991;63(2):139-46.

44. DeMichele A, Harding JJ, Telli ML, Munster P, McKay R, lliopoulos O, et al., editors. Phase 1 study of CB-839, a small molecule inhibitor of glutaminase (GLS) in combination with paclitaxel (Pac) in patients (pts) with triple negative breast cancer (TNBC). San Antonio: San Antonio Breast Cancer Symposium; 2016.

45. Budczies J, Pfitzner BM, Gyorffy B, Winzer KJ, Radke C, Dietel M, et al. Glutamate enrichment as new diagnostic opportunity in breast cancer. Int J Cancer. 2015;136(7):1619-28.

46. Haukaas TH, Euceda LR, Giskeødegård GF, Lamichhane S, Krohn M, Jernström S, et al. Metabolic clusters of breast cancer in relation to geneand protein expression subtypes. Cancer Metab. 2016;4(1):12.

47. Aure MR, Vitelli V, Jernstrom S, Kumar S, Krohn M, Due EU, et al. Integrative clustering reveals a novel split in the luminal A subtype of breast cancer with impact on outcome. Breast Cancer Res. 2017:19(1):44.

48. Kim S, Kim DH, Jung WH, Koo JS. Expression of glutamine metabolismrelated proteins according to molecular subtype of breast cancer. Endocr Relat Cancer. 2013;20(3):339-48.

49. Grinde MT, Moestue SA, Borgan E, Risa O, Engebraaten O, Gribbestad IS. $13 \mathrm{C}$ high-resolution-magic angle spinning MRS reveals differences in glucose 
metabolism between two breast cancer xenograft models with different gene expression patterns. NMR Biomed. 2011;24(10):1243-52.

50. Yuneva MO, Fan TW, Allen TD, Higashi RM, Ferraris DV, Tsukamoto T, et al. The metabolic profile of tumors depends on both the responsible genetic lesion and tissue type. Cell Metab. 2012;15(2):157-70.

51. Reeds PJ. Dispensable and indispensable amino acids for humans. J Nutr. 2000;130(7):1835S-40S

Ready to submit your research? Choose BMC and benefit from:

- fast, convenient online submission

- thorough peer review by experienced researchers in your field

- rapid publication on acceptance

- support for research data, including large and complex data types

- gold Open Access which fosters wider collaboration and increased citations

- maximum visibility for your research: over $100 \mathrm{M}$ website views per year

At $\mathrm{BMC}$, research is always in progress.

Learn more biomedcentral.com/submissions 